\title{
Demonstrations Track of the 25th International Joint Conference on Artificial Intelligence
}

\author{
Carlos Linares López \\ Computer Science Department, Universidad Carlos III de Madrid, Spain \\ E-mail: carlos.linares@uc3m.es
}

There is a valuable body of research in Artificial Intelligence as witnessed by the large number of contributions to different journals and scientific conferences. This work, continued unceasingly for various decades, has given rise to a significant number of applications in fields other than Artificial Intelligence (e.g., medicine, communications, security, etc.) and, also, to assist in real-life situations - e.g., robots, unmanned vehicles (either terrestrial or aerial), autonomous assistant agents, recommender systems, etc. Indeed, the interest in applications of Artificial Intelligence has recently reached unprecedented peaks from the general public.

IJCAI is the International Joint Conference on Artificial Intelligence, the main international gathering of researchers in AI, and a reference conference in the world in all fields of Artificial Intelligence. The 25th International Joint Conference on Artificial Intelligence was held in New York City (United States) from July 9 to July 15, 2016, and it offered also a wide variety of satellite events. The Demonstration Track was held for the first time and it has been re-edited in consecutive editions of the conference since then.
The Demonstrations Track provides a unique opportunity in this regard. Its primary goals are: first, to provide a good framework for exchanging ideas between theory and practice; secondly, to showcase the applicability of different AI technologies to real-world problems.

The Demonstration Track received a record number of 91 submissions on different topics from fields such as: Computer Vision, Genetic algorithms, Knowledgebased systems, Learning algorithms, Natural Language Processing, Planning and Robotics. All submissions accepted to the Demonstration Track were invited to submit a larger version of their research to AI Communications which underwent a thorough reviewing process.

This track in AI Communications gives space to research work based on the best works accepted in the Demonstrations Track of the 25th IJCAI conference explicitly submitted for the Demonstrations Track, with various applications in Planning, Knowledge Engineering, Natural Language Processing, Semantic Web, Ontologies, Data Mining, Neural Networks and Deep Learning. 